TITLE:

\title{
Application of beam emission spectroscopy to NBI plasmas of Heliotron J
}

\section{AUTHOR(S):}

Kobayashi, S.; Kado, S.; Oishi, T.; Kagawa, T.; Ohshima, S.; Mizuuchi, T.; Nagasaki, K.; ... Takeuchi, M.; Toushi, K.; Sano, F.

\section{CITATION:}

Kobayashi, S.... [et al]. Application of beam emission spectroscopy to NBI plasmas of Heliotron J. REVIEW OF SCIENTIFIC INSTRUMENTS 2010, 81(10): 10D726.

\section{ISSUE DATE:}

2010-10

\section{URL:}

http://hdl.handle.net/2433/147219

\section{RIGHT:}

Copyright 2010 American Institute of Physics. This article may be downloaded for personal use only. Any other use requires prior permission of the author and the American Institute of Physics. The following article appeared in REVIEW OF SCIENTIFIC INSTRUMENTS81, 10D726 (2010) and may be found at 


\title{
Application of beam emission spectroscopy to NBI plasmas of Heliotron $\mathrm{J}^{\mathrm{a})}$
}

\author{
S. Kobayashi, ${ }^{1, b)}$ S. Kado, ${ }^{2}$ T. Oishi, ${ }^{3}$ T. Kagawa, ${ }^{4}$ S. Ohshima, ${ }^{1}$ T. Mizuuchi, ${ }^{1}$ \\ K. Nagasaki, ${ }^{1}$ S. Yamamoto, ${ }^{1}$ H. Okada, ${ }^{1}$ T. Minami, ${ }^{1}$ S. Murakami, ${ }^{5}$ H. Y. Lee, ${ }^{4}$ T. Minami, ${ }^{4}$ \\ Y. Nakamura, ${ }^{4}$ K. Hanatani, ${ }^{1}$ S. Konoshima, ${ }^{1}$ M. Takeuchi, ${ }^{1}$ K. Toushi, ${ }^{1}$ and F. Sano ${ }^{1}$ \\ ${ }^{1}$ Institute of Advanced Energy, Kyoto University, Uji, Kyoto 611-0011, Japan \\ ${ }^{2}$ School of Engineering, The University of Tokyo, Bunkyo, Tokyo 113-8656, Japan \\ ${ }^{3}$ Nagoya University, Chikusa-ku, Nagoya 464-8603, Japan \\ ${ }^{4}$ Graduate School of Energy Science, Kyoto University, Uji, Kyoto 611-0011, Japan \\ ${ }^{5}$ Graduate School of Engineering, Kyoto University, Kyoto, Kyoto 606-8501, Japan
}

(Presented 18 May 2010; received 19 May 2010; accepted 5 September 2010; published online 22 October 2010)

\begin{abstract}
This paper describes the application of the beam emission spectroscopy (BES) to Heliotron J, having the nonsymmetrical helical-magnetic-axis configuration. The spectral and spatial profile of the beam emission has been estimated by the numerical calculation taking the collisional excitation processes between plasmas (electrons/ions) and beam atoms. Two sets of the sightlines with good spatial resolution are presented. One is the optimized viewing chords which have 20 sightlines and observe the whole plasma region with the spatial resolution $\Delta \rho$ less than \pm 0.055 using the newly designed viewing port. The other is 15 sightlines from the present viewing port of Heliotron $\mathbf{J}$ for the preliminary measurement to discuss the feasibility of the density fluctuation measurement by BES. The beam emission has been measured by a monochromator with a CCD camera. A good consistency has been obtained between the spectral profiles of the beam emission measured by the monochromator and the beam emission spectrum deduced by the model calculation. An avalanche photodiode with an interference filter system was also used to evaluate the signal-to-noise $(\mathrm{S} / \mathrm{N})$ ratio of the beam emission in the present experimental setup. The modification of the optical system is being planned to improve the $\mathrm{S} / \mathrm{N}$ ratio, which will enable us to estimate the density fluctuation in Heliotron J. () 2010 American Institute of Physics. [doi:10.1063/1.3495787]
\end{abstract}

\section{INTRODUCTION}

The plasma turbulent transport has long been an important subject in the magnetically confined fusion research. The technique of beam emission spectroscopy (BES), being the spectral measurement of the Doppler-shifted light emission from the excitation reaction between the hydrogen or deuterium neutral beam atoms and plasmas, has been developed in tokamak and helical devices, ${ }^{1-4}$ which have been a great advantage for understanding the physics of MHD activities and long-wavelength plasma turbulence. One of the key subjects for the development in the beam emission spectroscopy is to minimize the radial extent of the observation region which is determined by the intersection of the neutral beam and sightline. In the case of BES for the helical-magnetic-axis heliotron configuration, it is difficult to improve the spatial resolution because the magnetic axis and the flux surface of the helical-magnetic-axis heliotron configuration have threedimensional structure. Therefore, it is important to optimize the sightlines and establish a generally applicable way to the local measurement of the neutral-beam-based diagnostics

\footnotetext{
a) Contributed paper, published as part of the Proceedings of the 18th Topical Conference on High-Temperature Plasma Diagnostics, Wildwood, New Jersey, May 2010.

${ }^{b)}$ Electronic mail: kobayashi@iae.kyoto-u.ac.jp.
}

(BES, charge-exchange recombination spectroscopy, and motional-Stark effect) in the helical-magnetic-axis configurations.

In this study, we describe the application of BES to Heliotron $\mathrm{J}$, having the helical-magnetic-axis heliotron configuration $(R / a=1.2 \mathrm{~m} / 0.2 \mathrm{~m})$ with $L / M=1 / 4$ helical winding coil. ${ }^{5}$ The numerical calculation was carried out to estimate the spatial and spectral profile of the beam emission deduced from the collisional excitation model between the electrons/ ions and the neutral beam atoms in plasmas ${ }^{1,4,6}$ deduced from the beam trajectory analysis using Monte Carlo method. ${ }^{7}$ In order to discuss the feasibility of the density fluctuation measurement by BES in Heliotron J, a preliminary measurement of the beam emission was carried out and the results were compared with the model calculation. The optimization of the sightlines for the local measurement of the beam emission and the improvements in the signal-to-noise ratio are discussed.

\section{BEAM EMISSION SPECTROSCOPY FOR HELIOTRON J}

In Heliotron J, two tangential NBI beamlines (BL1:counter- and BL2:cogoing directions) have been installed with maximum acceleration voltage and power of 30 $\mathrm{kV}$ and $0.7 \mathrm{MW}$. Each beamline has two (upper and lower) bucket-type ion sources whose depression and elevation 
angle are set to be $3.5^{\circ}$ to the equatorial plane. The beamlet divergence is estimated to be $1.2^{\circ}$ by beam calibration experiments.

In the case of the line-emission of hydrogen atom from $n=3$ to $n=2$ state (i.e., $\mathrm{H}_{\alpha}$ line-emission; $h \nu=3.03$ $\times 10^{-19} \mathrm{~J}$ ), the intensity of the beam emission $I_{\mathrm{BES}}$ of the sightline $l$ is mainly determined by the collisional excitation process between the neutral beam hydrogen atoms and the background plasmas (electrons and hydrogen ions), ${ }^{1,4,6}$

$$
\begin{aligned}
I_{\mathrm{BES}}= & \int d l \frac{A_{32}}{A_{32}+A_{31}}\left(n_{i} n_{\text {beam }} \sigma_{i} v_{\text {beam }}+n_{e} n_{\text {beam }}\right. \\
& \left.\times\left\langle\sigma_{e}\left|v_{\text {beam }}-v_{e}\right|\right\rangle\right) h \nu S \Delta \Omega / 4 \pi,
\end{aligned}
$$

where $A_{n m}$ is the transition probability from the $n$ to the $m$ state, while $n_{i}, n_{e}, n_{\text {beam }}, v_{e}$, and $v_{\text {beam }}$ are the ion, electron and the neutral beam densities, and the electron thermal and the beam velocities, respectively. The effect of the thermal velocity for the bulk ion is negligible because of the lower ion temperature $\left(T_{i}(0)<0.3 \mathrm{keV}\right)$ than the injected beam energy $\left(E_{b}=24-30 \mathrm{keV} \mathrm{\textrm {H } ^ { 0 }}\right.$ beam). Under the typical experimental condition of the NBI plasmas of Heliotron J, the cross section of the ion impact excitation $\sigma_{i}$ is $1.7 \times 10^{-17} \mathrm{~cm}^{2}$, while the effective cross section for electron impact excitation $\left\langle\sigma_{e}\left|v_{\text {beam }}-v_{e}\right|\right\rangle / v_{\text {beam }}$ is $2.3 \times 10^{-17} \mathrm{~cm}^{2}$ in the case of the electron temperature $T_{e}$ of $0.4 \mathrm{keV}$. $S$ and $\Delta \Omega / 4 \pi$ are the observation area along the sightline element $d l$ and the solid angle, respectively.

A numerical model calculation is carried out to investigate the favorable sightlines for the Heliotron $\mathrm{J}$ configuration. The beam density is deduced from the analysis of the neutral beam trajectory in the Heliotron J plasmas including the beam attenuation effects. ${ }^{7}$ The amount of the Dopplershift of the beam emission, defined by $v_{\text {beam }} / c \cos \left(\theta_{\mathrm{BES}}\right) \lambda_{\mathrm{H} \alpha}$, is also estimated using the result of the model calculation, where $c, \theta_{\mathrm{BES}}$, and $\lambda_{\mathrm{H} \alpha}$ are the light speed, the angle between sightline and neutral beam, and the wavelength of the $\mathrm{H}_{\alpha}$ line, respectively. The density and the temperature profiles are assumed to be parabolic one having core values of $n_{e}(0)=n_{i}(0)=3 \times 10^{19} \mathrm{~m}^{-3}$ and $T_{e}(0)=0.4 \mathrm{keV}$, respectively.

Two candidates for the BES sightlines are shown in Figs. 1 (a) and 1(b). One is for the whole plasma region measurement using a newly designed viewing port and the other is the feasible sightlines from the present viewing port to evaluate the potential of BES for the density fluctuation measurement in Heliotron J. The co- and counter-NBI beamlines (BL2 and BL1) are also shown in the figures. The chords for the whole plasma measurement have 20 sightlines with 10 $\mathrm{mm}$ pitch. The angle $\theta_{\mathrm{BES}}$ is $24^{\circ}$ and $30^{\circ}$ for the upper and lower ion sources of co-NBI (BL2) and $150^{\circ}$ and $156^{\circ}$ for them of counter-NBI (BL1), respectively. As shown in the figure, since the magnetic axis of Heliotron $\mathrm{J}$ has square shape from the top view and sinuslike one from the side view, the viewing chords are selected so as to be parallel to the magnetic axis and to observe the beam intersection region. ${ }^{8,9}$ In the case of the sightlines using the present viewing port, 15 sightlines are aligned at $7 \mathrm{~mm}$ radial separation intervals. $\theta_{\mathrm{BES}}$ is $22^{\circ}$ and $28^{\circ}$ for $\mathrm{BL} 2$ and $155^{\circ}$ and $162^{\circ}$ for (a)

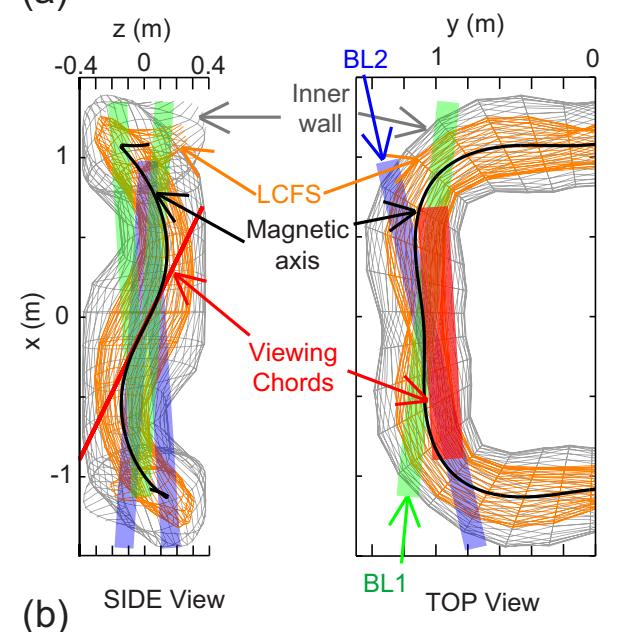

(b)

Rev. Sci. Instrum. 81, 10D726 (2010)
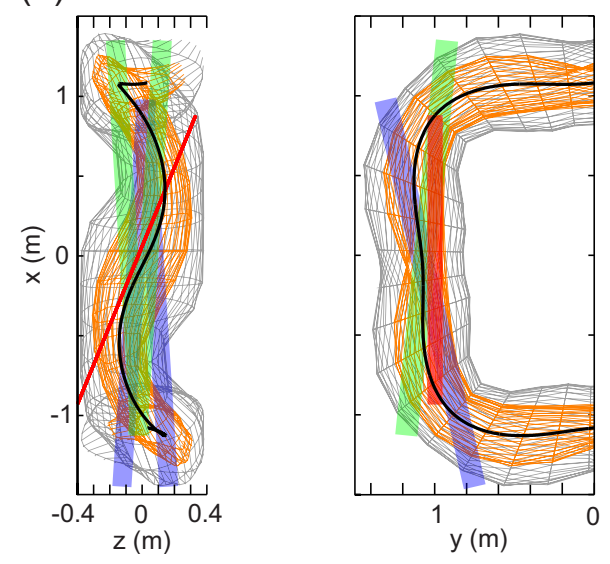

FIG. 1. (Color online) Schematic view of the BES viewing chords, (a) 20 sightlines for the whole plasma measurement using newly designed viewing port and (b) 15 sightlines from the present viewing port in Heliotron J. The co- and counter-NBI beamlines are also shown in the figures.

BL1. The optical systems of the optimized sightlines shown in Fig. 1(a) are being prepared. In this paper, we measured the beam emission using the sightlines shown in Fig. 1(b).

Figures 2(a) and 2(b) show the radial profile of the beam emission intensity estimated by Eq. (1) for the sightlines as shown in Figs. 1(a) and 1(b), respectively. Sightlines shown in Fig. 1(a) can observe the whole plasma region $(0<\rho$ $<1)$. The sightline has the spatial resolution $\Delta \rho$, determined from the half width at $1 / e$ height of the beam emission intensity, less than \pm 0.05 except for the sightlines of nos. 2 and 3 , because the sightlines are selected to minimize the spatial resolution at the plasma core. The spatial resolution in absolute units was $\pm 8 \mathrm{~mm}$. The spatial resolution $\Delta \rho$ for the sightline nos. 2 and 3 is slightly larger than \pm 0.05 , but smaller than \pm 0.055 . The spatial pitch between sightlines $\Delta x$ is $10 \mathrm{~mm}$, which yields the Nyquist wavenumber, $k_{\mathrm{N}}$ $=\pi / \Delta x$, of $=3.1 \mathrm{rad} \mathrm{cm}^{-1}$. The measurable wavenumber range $k_{\mathrm{N}}, \rho_{\mathrm{S}}$ is estimated to be smaller than 0.6 in the case that the ion Lamor radius $\rho_{\mathrm{S}}$ is $0.2 \mathrm{~cm}$ in the standard parameter of Heliotron $\mathrm{J}$ plasmas (the ion temperature of 0.3 $\mathrm{keV}$ and the magnetic field strength of $1.25 \mathrm{~T}$ ). The intensity of beam emission has a peaked profile which reflects the profiles of the neutral beam and plasma densities. The observation region shown in Fig. 1(b), on the contrary, is in the 

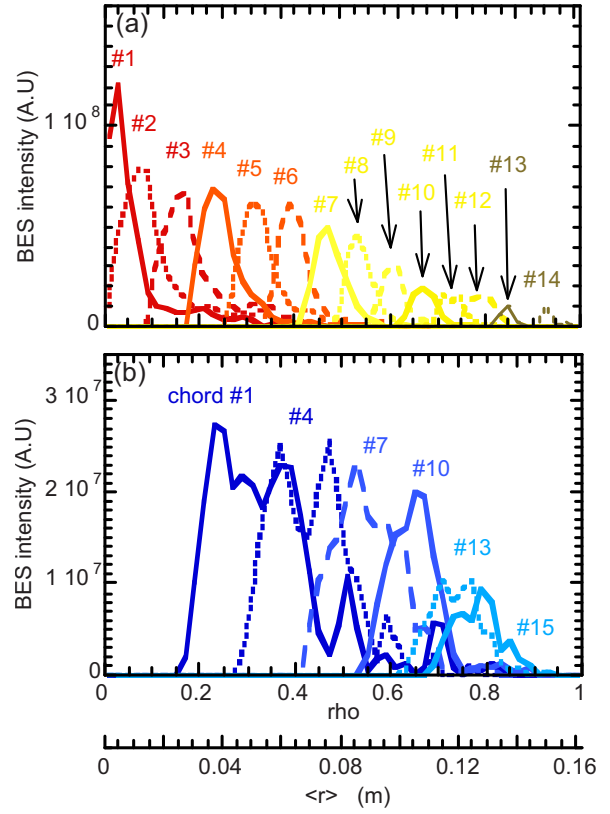

FIG. 2. (Color online) Radial profile of the beam emission intensity deduced by the numerical calculation in two cases: (a) for the sightlines for the whole plasma measurement using newly designed viewing port and (b) for the sightlines from the present viewing port of Heliotron J.

range of $\rho=0.3-0.8$ with $\Delta \rho$ of \pm 0.1 ( $\pm 16 \mathrm{~mm}$ in absolute units). A relative poor spatial resolution is owing to the location of the present viewing port.

\section{RESULTS AND DISCUSSIONS}

To discuss the feasibility of the density fluctuation measurement by BES in Heliotron $\mathrm{J}$, the optical system with fifteen sightlines as shown in Fig. 1(b) was installed into Heliotron $\mathrm{J}$ using an array of the optical fiber with each core diameter of $0.2 \mathrm{~mm}$ and NA of 0.2 . The beam emission is transferred to a monochromator (Czerny-Turner grating, $\mathrm{f}=400 \mathrm{~mm}, \mathrm{~F} 2.8$ ) equipped with a CCD camera to obtain the spectral profile of the beam emission. For the trial for the high-frequency BES measurement, the beam emission is also measured by an avalanche photodiode (APD) (Hamamatsu C4777-8643) through an interference filter with the condenser lens system. The $1 \mathrm{MHz}$ digitizer is used to acquire the APD signal. The solid angle and observation volume of this optical system are $\Delta \Omega=1.1 \times 10^{-5}$ sr and $\int S d l$ $=29 \mathrm{~cm}^{3}$, respectively.

Figure 3 shows the spectral profile of the beam emission at $\rho=0.4$ for the counter-NBI (BL1) plasmas at the lineaveraged electron density $\bar{n}_{e}$ of $1 \times 10^{19} \mathrm{~m}^{-3}$. The acceleration voltage of the hydrogen beam $\left(E_{b}\right)$ was $27 \mathrm{kV}$. The three Doppler-shifted components of the beam emission (full, half, and third energy components) are clearly separated without strong impurity-line-emissions. The spectral profile for the full energy beam component was compared with the model calculation described in the previous section. The split of the beam emission is owing to the difference of the observation angle between the upper and lower ion sources of NBI. A good consistency between the experimental result and the model calculation is found assuming a spectral broadening of $0.1 \mathrm{~nm}$ which is due to the instrument function of spectrom-

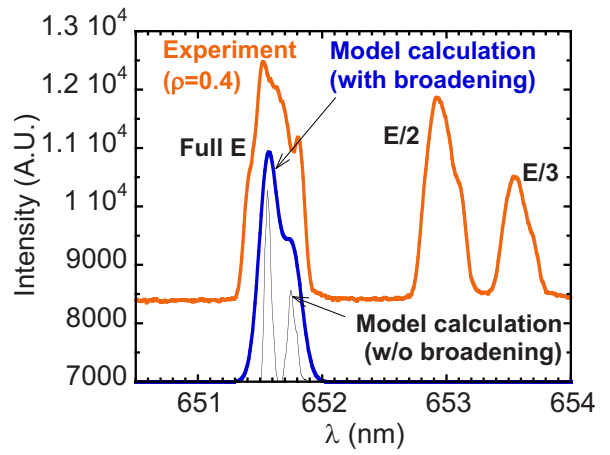

FIG. 3. (Color online) Spectral profiles of the beam emission deduced by the numerical calculation and measured by the monochromator.

eter and Zeeman effect. Note that the numerical analysis show that the beam emission intensity by the electron impact excitation was estimated to be 1.4-1.5 times higher than that for the ion because of the relatively low electron temperature $(\sim 0.4 \mathrm{keV})$ in the NBI plasmas of Heliotron J.

Figures 4(a)-4(d) show the time evolution of the plasma parameters and the beam emission intensity using the fast sampling $(1 \mathrm{MHz})$ system. The plasma was initiated by the $70 \mathrm{GHz}$ electron cyclotron heating $(\mathrm{ECH})$ and additionally heated by the co- and counter-NBI $\left(E_{b}=27 \mathrm{kV}\right)$. An interference filter with the center wavelength of $651.9 \mathrm{~nm}$ and the pass band of $1 \mathrm{~nm}$ at full width at half maximum was used to pass the beam emission of the full energy component. As shown in Fig. 4(d), the beam emission intensity started to rise up after turning-on the counter-NBI. In this discharge, $\mathrm{CH}_{4}$ gas was additionally introduced at $t=228 \mathrm{~ms}$ [see Fig. 4(c)], resulting increase in the stored energy and the lineaveraged electron density. The change in the beam emission intensity was observed in response to the density change. Since the ratio of the beam emission intensity to the noise intensity, regarding as the signal-to-noise $(\mathrm{S} / \mathrm{N})$ ratio of the

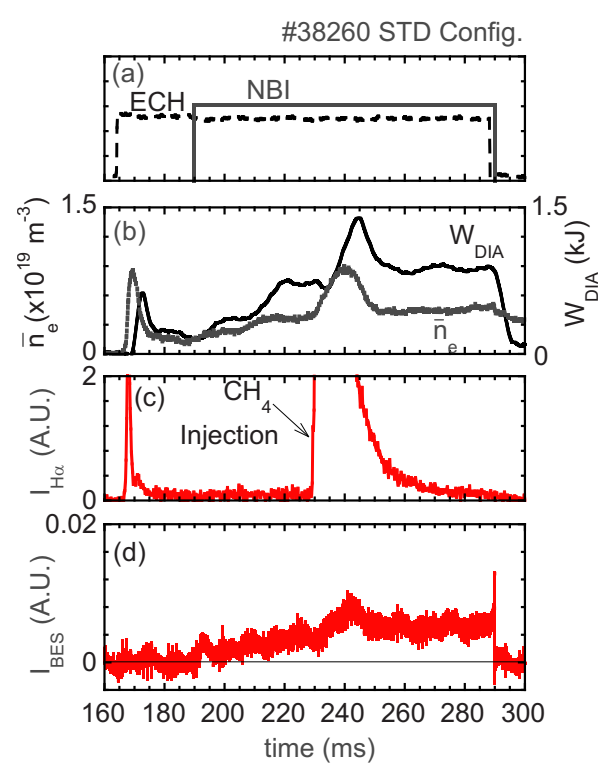

FIG. 4. (Color online) Time evolution of plasma parameters obtained in NBI plasmas of Heliotron J, (a) waveform of ECH and NBI, (b) the stored energy $\left(\mathrm{W}_{\text {DIA }}\right)$ and the line-averaged electron density $\left(\bar{n}_{e}\right)$, (c) the $\mathrm{H}_{\alpha}$ lineemission intensity, and (d) the beam emission intensity measured by APD. 
present BES system, was about 2 at $\bar{n}_{e}$ of $1 \times 10^{19} \mathrm{~m}^{-3}$, the improvement in the $\mathrm{S} / \mathrm{N}$ ratio more than 100 is required to evaluate the density fluctuation $(\tilde{n} / n \sim 1 \%)$.

In order to improve the $\mathrm{S} / \mathrm{N}$ ratio, we are installing the new-diagnostic port and modifying the optical system to put the designed sightlines with good spatial resolution into practice. To use the optical fiber array with core diameter of $0.8 \mathrm{~mm}$ and NA of 0.22 , the solid angle and the observation volume for the designed sightlines shown in Fig. 1(a) will increase up to $\Delta \Omega=5.7 \times 10^{-5} \mathrm{sr}$ and $\int S d l=60 \mathrm{~cm}^{3}$, respectively. Moreover, the spectral transmission profile of the interference filter will be modified in conjunction with the improvement in the throughput of the condenser lens system by use of the camera lenses. These modifications will provide increase in the $\mathrm{S} / \mathrm{N}$ ratio about 100 , which will enable us to measure the density fluctuation by BES in Heliotron J.

\section{ACKNOWLEDGMENTS}

One of the authors (S.K.) would like to acknowledge Professor Okamura, Professor Ida, and Dr. Nakano at NIFS for their collaboration of our research. The authors wish to thank the members of the Heliotron $\mathrm{J}$ group for their support in the experiments. This work was supported by NIFS/NINS under the NIFS Collaborative Research Program (Grant Nos. NIFS04KUHL005, NIFS09KUHL025, etc.) and under a project sponsored by the Formation of International Network for Scientific Collaborations. This work was partly supported by Grant-in-Aid for Young Scientists A No. 20686061.

${ }^{1}$ R. J. Fonck, P. A. Duperrex, and S. F. Paul, Rev. Sci. Instrum. 61, 3487 (1990).

${ }^{2}$ R. D. Durst, R. J. Fonck, G. Cosby, H. Evensen, and S. F. Paul, Rev. Sci. Instrum. 63, 4907 (1992).

${ }^{3}$ G. McKee, R. Ashley, R. Durst, R. Fonck, M. Jakubowski, K. Tritz, K. Burrell, C. Greenfield, and J. Robinson, Rev. Sci. Instrum. 70, 913 (1999).

${ }^{4}$ T. Oishi, S. Kado, M. Yoshinuma, K. Ida, S. Tanaka, and S. Okamura, J. Plasma Fusion Res. 6, 449 (2004); T. Oishi, Ph.D. thesis (in Japanese), The University of Tokyo, 2006.

${ }^{5}$ F. Sano, T. Mizuuchi, K. Kondo, K. Nagasaki, H. Okada, S. Kobayashi, K. Hanatani, Y. Nakamura, S. Yamamoto, Y. Torii, Y. Suzuki, H. Shidara, M. Kaneko, H. Arimoto, T. Azuma, J. Arakawa, K. Ohashi, M. Kikutake, N. Shimazaki, T. Hamagami, G. Motojima, H. Yamazaki, M. Yamada, H. Kitagawa, T. Tsuji, H. Nakamura, S. Watanabe, S. Murakami, N. Nishino, M. Yokoyama, Y. Ijiri, T. Senju, K. Yaguchi, K. Sakamoto, K. Tohshi, and M. Shibano, Nucl. Fusion 45, 1557 (2005).

${ }^{6}$ R. K. Janev, D. Reiter, and U. Samm, FZJ Report No. Juel-4105, 2003.

${ }^{7}$ S. Murakami, N. Nakajima, and M. Okamoto, Trans. Fusion Technol. 27, 256 (1995).

${ }^{8}$ S. Kado (private communication), 2008.

${ }^{9}$ S. Kobayashi et al. J. Plasma Fusion Res. SERIES 9, 59 (2010). 